\title{
Arteritis de Takayasu con estenosis severa de ramas pulmonares principales
}

\author{
Pablo Flores ${ }^{1}$, Nicolás González ${ }^{2}$, Carlos Astudillo ${ }^{3}$. \\ 1. Médico internista, Unidad de paciente crítico del Hospital Carlos Van Buren de Valparaíso. \\ 2. Residente de Medicina interna de la Universidad de Valparaíso, Servicio de Medicina interna del Hospital Carlos Van Buren de Valparaíso. \\ 3. Cardiólogo, Servicio de Cardiología del Hospital Carlos Van Buren de Valparaíso.
}

*Declaramos no tener conflictos de interés.

\section{Takayasu's arteritis with severe stenosis of main pulmonary artery branches}

A 40-year-old woman consulted for progressive dyspnea. Physical examination revealed systolic murmurs in the upper thoracic area and asymmetric pulses in the upper extremities. Echocardiography revea- led a 117-mmHg systolic gradient across the tricuspid valve and CT angiography revealed severe stenosis of pulmonary artery branches. A discussion of the disease is included.

\section{Correspondencia:}

Dr. Pablo Flores Fernández

Dirección: San Ignacio N 725, Hospital Carlos Van Buren, Valparaíso

Cel.: 56-322364000, 56-942889594

pablo.flofer@gmail.com 


\section{Introducción:}

La Arteritis de Takayasu es una vasculitis crónica de etiología desconocida que afecta preferentemente grandes vasos, principalmente la aorta y sus ramas ${ }^{1}$. Tiene distribución mundial, pero presenta mayor prevalencia en asiáticos ${ }^{2}$. La incidencia estimada en Estados Unidos y Europa es de 2-3 casos por millón de habitantes ${ }^{3}$, pero es desconocida en Chile.

Las lesiones vasculares iniciales en la Arteritis de Takayasu frecuentemente ocurren en la porción proximal o media de la arteria subclavia izquierda y a medida que progresa la enfermedad puede comprometer la arteria carótida común izquierda, arterias vertebrales, tronco braquiocefálico, porción proximal y media de arteria subclavia derecha, arteria carótida común derecha y aorta. También puede afectar la arteria pulmonar y sus ramas en el $50 \%$ de los casos, siendo poco frecuente la arteritis pulmonar aislada ${ }^{4}$. En cerca del $10 \%$ de los pacientes hay aneurismas en localizaciones variables ${ }^{5}$.

El compromiso de arteria pulmonar y sus ramas puede dar variada sintomatología, que raramente es la manifestación inicial o principal del cuadro clínico, implica un peor pronóstico de la enfermedad, adicionando importante morbimortalidad y su enfrentamiento representa un reto terapéutico.

A continuación, se presenta un caso clínico de un paciente con diagnóstico de Arteritis de Takayasu con compromiso severo de la arteria pulmonar y sus ramas principales. La sospecha diagnóstica se realizó mediante ecocardiografía transtorácica y posteriormente fue apoyado por tomografía computada. Se ilustra la importancia de la evaluación ecográfica de la válvula y arteria pulmonar, y se muestran cortes tomográficos axiales y sagitales evidenciando el extenso compromiso aórtico, de troncos supra aórticos y de las ramas principales de la arteria pulmonar. Además, se comentan las opciones terapéuticas intervencionistas disponibles para esta enfermedad.

\section{Caso clínico:}

Mujer de 40 años con antecedente de hipotiroidismo primario y usuaria de marcapasos bicameral DDD por bloqueo auriculoventricular de alto grado de reciente aparición. Acudió a consulta por cuadro de 6 meses de disnea progresiva hasta hacerse de moderados esfuerzos, asociado a baja de peso y astenia, sin otros síntomas agregados. Al examen físico destacó la presencia de un soplo proto-mesodiastólico en foco aórtico, pulsos periféricos asimétricos, siendo de menor amplitud en extremidad superior izquierda y extremidades inferiores, con

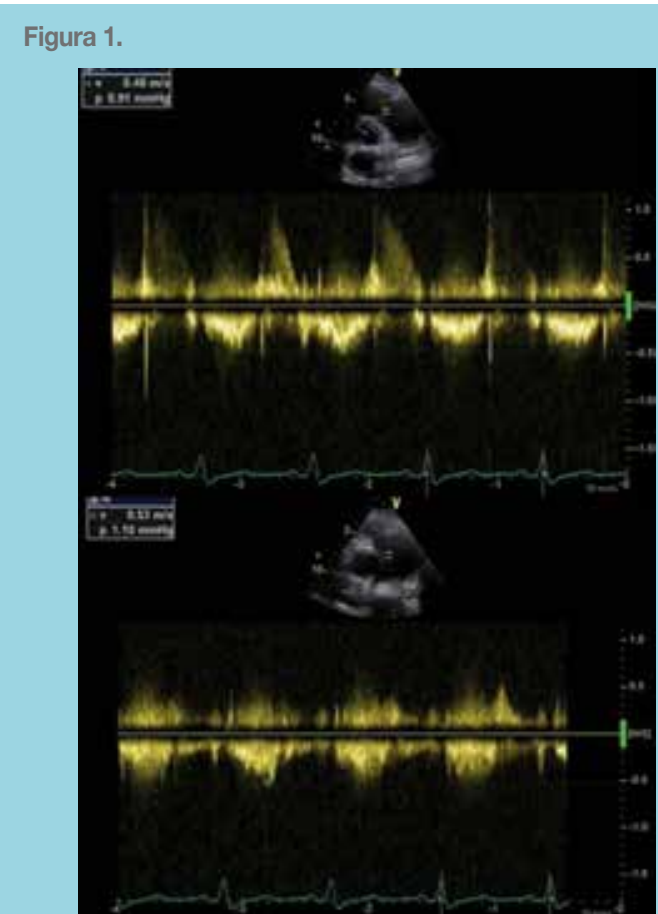

Arriba: Doppler pulsado a nivel de válvula pulmonar sin gradiente significativa. Abajo: Doppler pulsado a nivel de tronco de arteria pulmonar sin gradiente significativa.

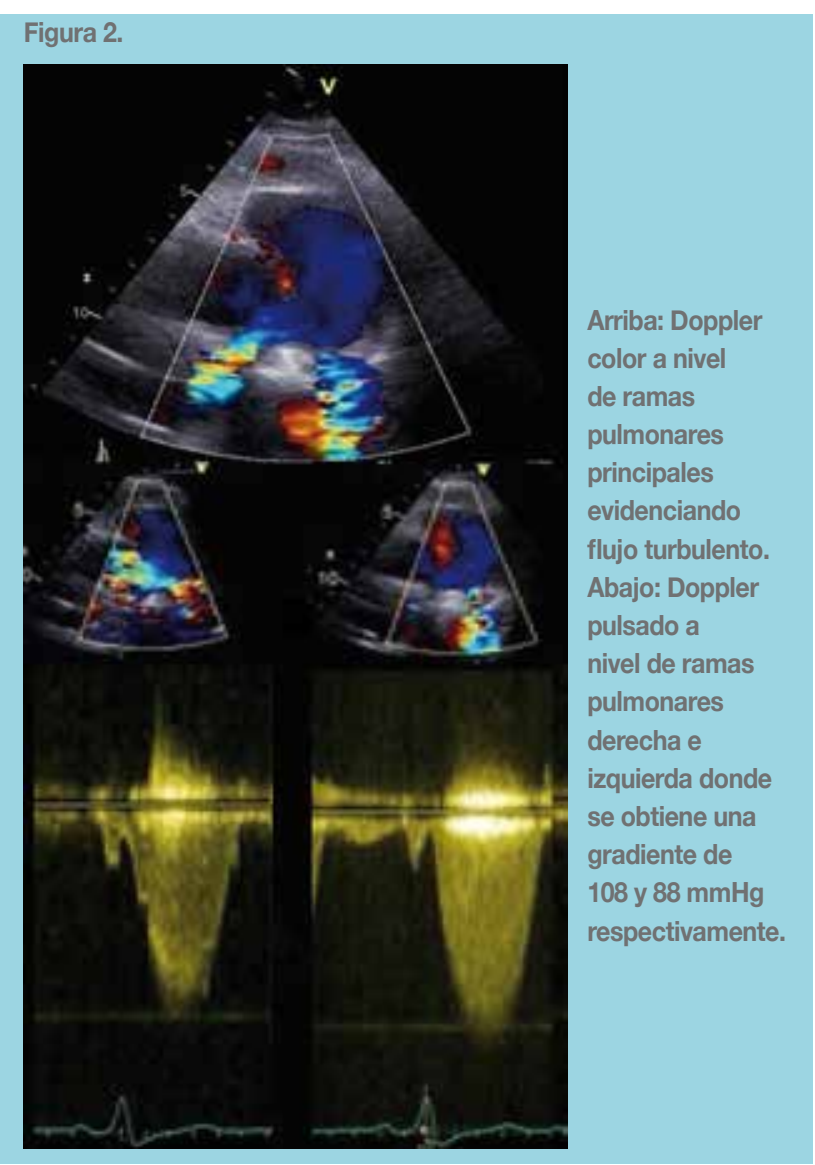



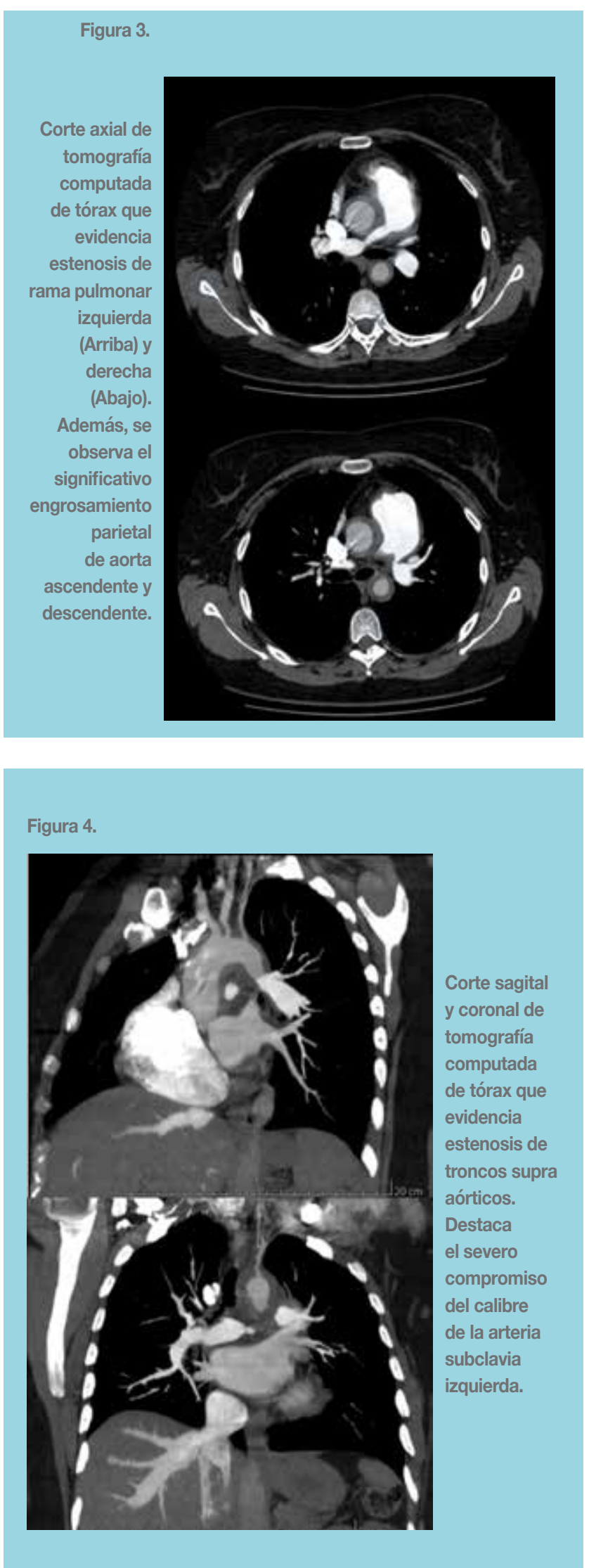

Figura 5

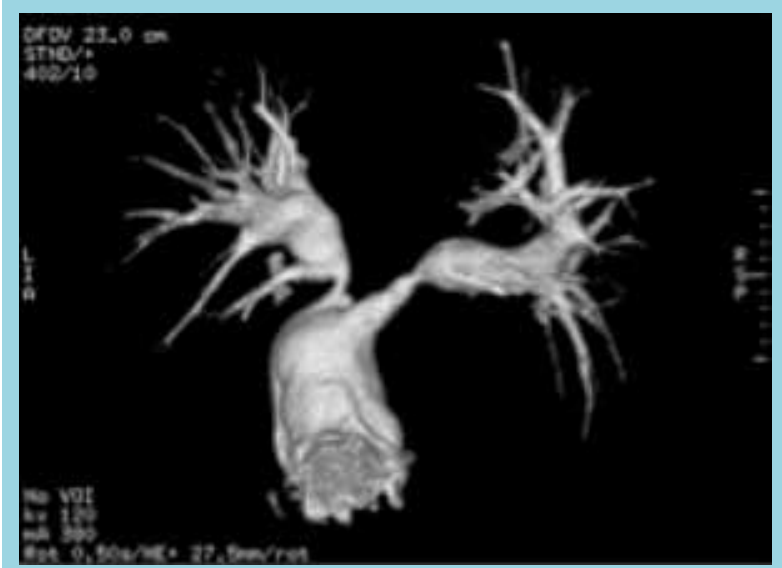

Reconstrucción tomográfica 3D de arteria pulmonar. Se observa el severo grado de estenosis que presentan sus ramas principales.

una diferencia significativa $(>10 \%)$ de presión arterial entre extremidades superiores. No había signos de congestión pulmonar, las yugulares no se encontraban distendidas, no había hepatomegalia ni edema periférico. Sumado a lo anterior se pesquisó soplo sistólico dorsal a la búsqueda dirigida. El laboratorio general evidenció anemia normocítica normocrómica, elevación de reactantes de fase aguda con PCR $26 \mathrm{mg} / \mathrm{dL}$ y VHS $68 \mathrm{~mm} /$ hr e hipoalbuminemia. Un ECG mostró ritmo de marcapasos con estimulación auricular y ventricular, sin signos de sobrecarga ventricular derecha. El ecocardiograma transtorácico mostró fracción de eyección de 57\%, cavidades cardíacas de tamaño normal, e hipertrofia del ventrículo derecho con TAPSE de $22 \mathrm{~mm}$. Había regurgitación aórtica moderada y el doppler continuo a nivel tricuspídeo demostró gradiente de $117 \mathrm{mmHg}$. Mediante doppler pulsado se demostró que la válvula pulmonar y tronco de la arteria pulmonar no presentaban gradiente significativa, pero que existía una estenosis severa a nivel de ambas ramas pulmonares principales, con una gradiente de $88 \mathrm{mmHg}$ para la rama derecha y $108 \mathrm{mmHg}$ para la rama izquierda. El ecocardiograma transesofágico confirmó los hallazgos descritos y mostró una reducción del diámetro de la aorta descendente luego del nacimiento de la arteria subclavia izquierda, con una gradiente estimada de $56 \mathrm{mmHg}$. Con los hallazgos anteriores se solicitó una tomografía de tórax que mostró tronco de arteria pulmonar dilatado, con estenosis severa de las ramas pulmonares principales y, además, evidencia estrechamiento luminal y engrosamiento parietal de aorta ascendente, cayado y espe- 
cialmente de aorta descendente con un grosor parietal de $7 \mathrm{~mm}$, que explica el gradiente de aorta descendente encontrado en el ecocardiograma. También se aprecia estenosis significativa de ramas supra aórticas, especialmente de arteria subclavia izquierda que es filiforme. Un estudio complementario permitió descartar otras etiologías inmunológicas e infecciosas. El VDR fue no reactivo y las pruebas treponémicas (MHA-TP) negativas. Se formuló el diagnóstico de Arteritis de Takayasu con estenosis severa de ramas pulmonares principales, y se inició prednisona a dosis de $1 \mathrm{mg} / \mathrm{kg} /$ día con buena respuesta clínica inicial, mejoría de capacidad funcional y disnea. El ecocardiograma transtorácico de control no mostró variación. Se mantiene en seguimiento clínico periódico y se están evaluando las posibilidades de terapia intervencionista endovascular dado su compromiso severo de arteria pulmonar.

\section{Discusión:}

La Arteritis de Takayasu corresponde a una enfermedad poco frecuente y grave que connota un pronóstico desfavorable para nuestro paciente y su familia. Se sabe que la sobrevida de pacientes con la enfermedad alcanza el $83,1 \%$ a 5 años de efectuado el diagnóstico, pero no hay datos sobre la calidad de vida asociada ${ }^{2}$. Es esperable que el compromiso de arteria pulmonar agregue morbimortalidad adicional a la conocida.

En el $80-90 \%$ de los casos de Arteritis de Takayasu se trata de mujeres jóvenes con una edad de inicio entre los 10-40 años ${ }^{3,6}$. El compromiso de arteria pulmonar es más frecuente en menores de 30 años y raza asiática ${ }^{7}$. Con mayor frecuencia se ha reportado la afectación de la arteria pulmonar del lóbulo superior derecho ${ }^{8}$. Las lesiones patológicas en las arterias pulmonares se parecen a las observadas en las arterias sistémicas e incluyen inflamación, lesiones granulomatosas en las capas elástica, media y adventicia, a menudo acompañadas por formación de trombos y dilatación vascular ${ }^{9}$.

Las manifestaciones clínicas más frecuentes de la Arteritis de Takayasu son síntomas inespecíficos como fatiga, baja de peso y fiebre, que son más comunes en las fases tempranas de la enfermedad. Puede haber mialgias y artralgias en la mitad de los casos ${ }^{10}$. A medida que progresa la enfermedad aparecen síntomas y signos relacionados con la oclusión e insuficiencia vascular de los territorios afectados, como frialdad de extremidades, pulsos periféricos débiles, claudicación de brazos y extremidades inferiores, discrepancia en la presión arterial de extremidades, soplos vasculares como carotideo y subclavio, angor pectoris, síncope y síntomas neurológicos ${ }^{11}$. En cerca de la mitad de los casos se desarrolla hipertensión arterial por estenosis de arterias renales y disminución de la elasticidad de la aorta y sus ramas ${ }^{10}$. En casos avanzados puede encontrarse ulceras cutáneas isquémicas y

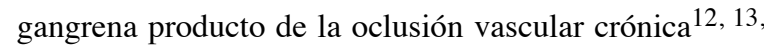
14. Los síntomas relacionados con el compromiso de la arteria pulmonar son poco frecuentes e incluyen dolor torácico, disnea, hemoptisis e hipertensión pulmonar. Hay muy pocos casos reportados de infartos pulmonares asociados ${ }^{9}$. Un estudio encontró arteritis pulmonar en 11 de 22 pacientes con arteritis de Takayasu, todos ellos se encontraban asintomáticos, pero la mayoría tenía alteraciones en el ECG y estudios de imágenes ${ }^{15}$.

No existen pruebas de laboratorio específicas para el diagnóstico. Actualmente la biopsia endovascular de un área estenótica ofrece la confirmación histológica, pero ésta no está ampliamente disponible y no se encuentra exenta de complicaciones ${ }^{16}$. Es por esto que el diagnóstico precisa de una excelente sospecha clínica y del apoyo imagenológico mediante tomografía computada, resonancia o angiografía que da muestra del estrechamiento del lumen arterial y engrosamiento de la pared vascular ${ }^{17}$. Ishikawa plantea que se puede aceptar el diagnóstico cuando se cumple el criterio obligado (Inicio de la enfermedad antes de los 40 años), más 2 criterios mayores, o 1 criterio mayor con 2 menores, o 4 criterios menores (Tabla 1) ${ }^{2}$. Creemos que este último modo de diagnóstico, sumado al descarte de otras causas, es asertivo y conveniente, por lo que sugerimos su uso en la práctica clínica, permitiendo un tratamiento precoz, que puede retrasar la evolución natural de la enfermedad.

\section{Tabla 1. Criterios diagnósticos de Arteritis de Takayasu según} Ishikawa.

Condición obligatoria: inicio de los síntomas antes de los 40 años.

Criterios mayores:

Compromiso de la porción media de la arteria subclavia izquierda. Compromiso de la porción media de la arteria subclavia derecha.

Criterios menores:

Hipertensión arterial.

VHS elevada.

Insuficiencia o anuloectasia aórtica.

Compromiso de la arteria pulmonar.

Compromiso de la carótida común izquierda en su porción media

Compromiso de la porción distal del tronco braquiocefálico.

Sensibilidad carotídea.

Compromiso variable de aorta torácica.

Compromiso variable de aorta abdominal. 
Si bien existe un tratamiento farmacológico descrito, principalmente basado en corticoides sistémicos, inmunosupresores y el propio de la insuficiencia cardíaca, éste logra la remisión de la enfermedad sólo en alrededor del $60 \%$ de los $\operatorname{casos}^{18}$. En la actualidad, existen opciones de terapias endovasculares que se pueden ofrecer durante la etapa de remisión a pacientes con enfermedad oclusiva severa con claudicación severa de extremidades, estenosis renal y síntomas neurológicos derivados de insuficiencia cerebrovascular, aunque este tratamiento sigue siendo controvertido en la literatura $^{19}$. La terapia endovascular es una opción válida sólo para un grupo muy seleccionado de pacientes, puesto que la mayoría presentan lesiones vasculares fibróticas y calcificadas que abarcan largos segmentos, que son difíciles de abordar y más propensas a complicaciones ${ }^{18}$. Dentro de los procedimientos endovasculares recientemente reportados se encuentran el uso de angioplastía con balón, con stents liberadores de drogas $^{20}$, con stents cubiertos y stent grafts ${ }^{21}$. En pequeños estudios se demuestra que las terapias endovasculares con stents podrían ser algo mejor que los procedimientos endovasculares tradicionales sin stents ${ }^{22}$.

Respecto a la terapia endovascular en la arteria pulmonar en pacientes con enfermedad de Takayasu, existe poca evidencia disponible y su seguridad no está establecida. Sin embargo, hay estudios que muestran beneficio con angioplastía pulmonar transluminal percutánea y angioplastía pulmonar (APTP) con y sin la implantación de stent $^{23}, 24,25,26$. Existe controversia acerca del mayor beneficio de una modalidad respecto a la otra. Es posible que la eficacia terapéutica de la angioplastía en esta patología este dado más por ablación de trombos intraluminales que por la propia dilatación ${ }^{27}$. Un estudio prospectivo que incluye a 14 pacientes con arteritis de Takayasu con compromiso de arteria pulmonar muestra beneficios posterior a APTP, reportando mejoría en clase funcional, aumento de la distancia de prueba de caminata de 6 minutos, mejoría en la saturación de oxígeno y disminución de presión media de arteria pulmonar con una tasa de complicaciones baja, 3 pacientes sufrieron isquemia pulmonar por reperfusión, falleciendo uno de ellos por esa causa ${ }^{28}$. Otro estudio retrospectivo que incluyó 6 pacientes con arteritis de Takayasu con compromiso de arteria pulmonar sometidos a APTP muestra mejoría de clase funcional y reducción de niveles de NT-proBNP posterior al procedimiento ${ }^{29}$. Se describe que el riesgo de sangrado extravascular postprocedimiento puede llegar hasta el $25.5 \% 27$.

En resumen, la terapia endovascular en pacientes con arteritis de Takayasu se encuentra en desarrollo actualmente, y puede ser una opción a considerar en pacientes con síntomas severos y compromiso estenótico severo de ramas arteriales, sin embargo, es importante destacar que son necesarios estudios adicionales para establecer adecuadamente el beneficio y eficacia de ésta intervención en este grupo de pacientes. 


\section{Referencias}

1. HATA A, NODA M, MORIWAKI R, NUMANO F. Angiographic findings of Takayasu arteritis: new classification. Int $\mathbf{J}$ Cardiol 1996; 54:S155.

2. ISHIKAWA K. Natural history and classification of occlusive thromboaortopathy (Takayasu's disease). Circulation 1978; 57:27.

3. AREND W, MICHEL B, BLOCH D, HUNDER G, CALABRESE L, EDWORTHY S, ET AL. The American College of Rheumatology 1990 criteria for the classification of Takayasu arteritis. Arthritis Rheum 1990; 33:1129.

4. ARÉVALO E, SÉNIOR J. Hipertensión pulmonar severa asociada a enfermedad de Takayasu. Iatreia 2014; $27: 4$

5. VALDÉS F, CARVAJAL S, MORALES B, GONZALEZ S, MERINO G, KRAMER S, ET AL. Aortitis de Takayasu y Aneurisma Torácico Complicado por Ruptura. Rev. Chil. de Cardiología. 1989; 8: 41-49.

6. DABAGUE J, REYES PA. Takayasu arteritis in Mexico: a 38year clinical perspective through literature review. Int J Cardiol 1996; 54:S103.

7. KERR G, HALLAHAN C, GIORDANO J, LEAVITT R, FAUCI A, ROTTEM M, ET AL. Takayasu arteritis. Ann Intern Med 1994; 120: 919-29.

8. HOTCHI M. Pathological studies on Takayasu arteritis. Heart Ves- sels Suppl 1992; 7: 11-7.

9. NARITA J, ITO S, TERADA M, SAITOH Y, IGARASHI K, NAKANO M, ET AL. Pulmonary artery involvement in Takayasu's arteritis with lung infarction and pulmonary aspergillosis. J Clin Rheumatol 2002; 8: 260-4.

10. MASON J. Takayasu arteritis-advances in diagnosis and management. Nat Rev Rheumatol. 2010; 6: 406-15.

11. SERRA R, BUTRICO L, FUGETTO F, CHIBIREVA M, MALVA A, DE CARIDI G, ET AL. Updates in Pathophysiology, Diagnosis and Management of Takayasu Arteritis. Ann Vasc Surg. 2016; 35: 210-25.

12. NAKABAYASHI K, KURATA N, NANGI N, MIYAKE H, NAGASAWA T. Pulmonary artery involvement as first manifestation in three cases of Takayasu arteritis. Int J Cardiol 1996; 54: S177.
13. RODRÍGUEZ-PLA A, DE MIGUEL G, LÓPEZ-CONTRERAS J, DE LLOBET J, LLAUGER J, DÍAZ C. Bilateral blindness in Takayasu's disease. Scand J Rheumatol 1996; 25:394.

14. KERR G, HALLAHAN C, GIORDANO J, LEAVITT R, FAUCI A, ROTTEM M, ET AL. Takayasu arteritis. Ann Intern Med 1994;120:919.

15. LUPI E, SANCHEZ G, HORWITZ S, GUTIERREZ E. Pulmonary artery involvement in Takayasu's arteritis. Chest 1975; 67: 69-74.

16. ISHIKAWA K. Diagnostic approach and proposed criteria for the clinical diagnosis of Takayasu's arteriopathy. J Am Coll Cardiol 1988; 12: 964-972.

17. KEENAN N, MASON J, MACEIRA A, ASSOMULL R, O'HANLON R, CHAN C, ET AL. Integrated cardiac and vascular assessment in Takayasu arteritis by cardiovascular magnetic resonance. Arthritis Rheum. 2009; 60: 3501.

18. WALLER R, AHMED A, PATEL I, LUQMANI R. Update on the classification of vasculitis. Best Pract Res Clin Rheumatol 2013; 27: 3-17.

19. JOSEPH G, GEORGE P, PATI P, CHANDY S. Feasibility of angioplasty and stenting for abdominal aortic lesions adjacent to previously stented visceral artery lesions in patients with Takayasu arteritis. Cardiovasc Intervent Radiol. 2007; 30: 293-6.

20. KAZIBUDZKI M, TEKIELI L, TRYSTUŁA M, PALUSZEK P, MOCZULSKI Z, PIENIĄŻEK P. New endovascular techniques for treatment of life-threatening Takayasu arteritis. Postepy Kardiol Interwencyjnej. 2016; 12: 171-174.

21. QURESHI M, MARTIN Z, GREENBERG R. Endovascular management of patients with Takayasu arteritis: stents versus stent grafts. Semin Vasc Surg. 2011; 24: 44-52.

22.MAVROGENI S, DIMITROULAS T, CHATZIIOANNOU S, KITAS G. The role of multimodality imaging in the evaluation of Takayasu arteritis. Semin Arthritis Rheum 2013; 42: 401-12.

23. YAMAZAKI I, ICHIKAWA Y, ISHII M, HAMADA T, KAJIWARA H. SURGICAL. Case of isolated pulmonary Takaya- 
su's arteritis. Circ J 2005; 69: 500-2.

24. SHIKATA H, SAKAMOTO S, UEDA Y, TSUCHISHIMA S, MATSUBARA T, NISHIZAWA H, ET AL. Reconstruction of bilateral branch pulmonary artery stenosis caused by Takayasu's aortitis. Circ J 2004; 68: 791-4.

25. NAKAJIMA N, MASUDA M, IMAMAKI M, ISHIDA A, TANABE N, KURIYAMA T. A case of pulmonary artery bypass surgery for a patient with isolated Takayasu pulmonary arteritis and a review of the literature. Ann Thorac Cardiovasc Surg 2007; 13: 267-71.

26. KASHIMA K, KAWASAKI D, YOTSUMOTO G, HATAKE $S$, YAMASHITA E, NAGAYOSHI S, ET AL. Rapid progression of aortic regurgitation with thoracic aortic aneurysm due to Takayasu arteritis associated with ulcerative colitis. Intern Med 2010; 49: 1007-11.

27. ROTHMAN A, LEVY D, SKLANSKY M, GROSSFELD P, AUGER W, AJAMI G, ET AL. Balloon angioplasty and stenting of multiple intralobar pulmonary arterial stenoses in adult patients. Catheter Cardiovasc Interv 2003; 58: 252-60.

28. KREUTZER J, LANDZBERG M, PREMINGER T, MANDELL V, TREVES S, REID L, ET AL. Isolated peripheral pulmonary artery stenoses in the adult. Circulation 1996; 93: 1417-23.

29. WANG X, DANG A, CHEN B, LV N, LIU Q. Takayasu arteritis associated pulmonary hypertension. J Rheumatol 2015; 42; 495-503. 